\title{
Multipath Fading Channel Optimization for Wireless Medical Applications
}

\author{
A.K.M Fazlul Haque, \\ Department of Computer Science \\ and Engineering, \\ Jahangirnagar University, Dhaka, \\ Bangladesh \\ akm_haque@yahoo. $\mathrm{com}^{1}$
}

\author{
Md. Hanif Ali \\ Department of Computer Science \\ and Engineering \\ Jahangirnagar University, Dhaka, \\ Bangladesh \\ hanif ju03eyahoo. $\mathrm{com}^{2}$
}

\author{
M Adnan Kiber \\ Department of Applied Physics, \\ Electronics and Communication \\ Engineering, \\ University of Dhaka, Dhaka, \\ Bangladesh
}

\begin{abstract}
In this paper, a new method has been proposed to eliminate the intersymbol interference (ISI) and interchannel interference (ICI) for discrete multitone/orthogonal frequency division multiplexing (DMT/OFDM) systems by considering Time to Live (TTL) of multipath channel fading, especially for wireless medical application. In this method, the existence time of the packet is considered as the maximum propagation time and when the packet is sent to the receiver, the down count of the TTL starts. The existence of the packet in a network depends on TTL in an Internet Protocol (IP) packet that tells a network router whether or not the packet has been in the network too long and should be discarded. The proposed structure prevents ICI with a preprocessing method that utilizes a particular time that is equal to the continuation time of the packet and removes ISI by canceling the replica at the receiver. The simulation results show that the proposed method reduces the BER/ISI better under mutipath fading environment than other existing system.
\end{abstract}

\section{Keywords: ISI, ICI, OFDM, TTL, Channel Fading}

\section{INTRODUCTION}

Recently, multipath fading channel is an imperative aspect for wireless communication, especially for biological data transmission. Fading is a serious impairing effect introduced by the radio wave's propagation through the channel and causes a big problem to the signal detection process at the receiver. When the signal experiences fading in the channel, both its envelope and phase will fluctuate over time. Where a coherent modulation scheme is concerned, the fading effects on the signal phase can seriously impair performance, unless some necessary measures are taken to compensate for them at the receiving end even at the cost of complexity of the receiver. In many cases, analysis of systems using such coherent modulation schemes assumes that the phase effects due to fading are perfectly corrected by the receiver randomness to the received signal [1]. So, it is needed to remedy the problem regarding this. There are many works on multipath channel fading [2-11]. Van Duc Nguyen et al considered the case of time-invariant channel for intersymbol and intercarrier interference [2]. Cheol-Jin Park et al described a new method to eliminate intersymbol interference (ISI) and interchannel interference (ICI) for discrete multitone/orthogonal frequency division multiplexing (DMT/OFDM) systems with insufficient cyclic prefix (CP) [3]. Yun Hee Kim et al analyzed the combined influence of the interchannel and intersymbol interferences, which result from the time variation and delay spread of mobile channels, on the performance of an orthogonal frequency-division multiplexing (OFDM) system [4]. Wei Zhong et al proposed two novel iterative cyclicity restoration (CR) schemes to efficiently cancel the interferences for OFDM-based wireless local area network (WLAN) systems [5]. Noriyoshi Suzuki et al proposed a new OFDM demodulation method in order to reduce the influence of the ISI and improved the bit error performance in the presence of multipath whose excess delays are longer than the Guard Interval [6]. Most of the works focused on to reduce the ISI and ICI and improved the bit error rate. Still the ISI and ICI tendency is being appeared in the existing work. In this method, the existence time of the packet is equal to the maximum propagation time and when the packet is sent to the receiver, the down count of the TTL starts. Time-to-Live (TTL) is a value in an Internet Protocol (IP) packet that tells a network router whether or not the packet has been in the network too long and should be discarded. When the TTL is equal to zero of a particular packet, the corresponding packet is discarded. In the proposed OFDM symbol the TTL value tells the network that how much time it travels in the network. Let, $20 \mathrm{~ms}$ is the time require for an OFDM symbol to travel from transmitter to receiver. When one symbol is reached to the receiver, the receiver is ready to receive the next symbol. When the next symbol is reached to the receiver the replica of the pervious symbol is also reached to the receiver. This is how the ISI happened. If the TTL is set in the OFDM symbol less than $25 \mathrm{~ms}$, after $25 \mathrm{~ms}$ the replicas of all symbols will be discarded and no ISI will be happen. In this paper, the ISI tendency has been eliminated mostly and an acceptable improvement of bit error rate has been introduced which is better than existing techniques. Simulation and results have been tested and verified using NS2.

\section{BACKGROUND THEORY}

\section{A. Capacity of fading channels}

The required theoretical background regarding the fading channel have been considered and discussed for the 
proposed method.

Consider the complex baseband representation of a flat fading channel:

$$
\mathrm{y}[\mathrm{m}]=\mathrm{h}[\mathrm{m}] \mathrm{x}[\mathrm{m}]+\mathrm{w}[\mathrm{m}]
$$

Where $\{\mathrm{h}[\mathrm{m}]\}$ is the fading process and $\{\mathrm{w}[\mathrm{m}]\}$ is noise. As before, the symbol rate is $\mathrm{W} \mathrm{Hz}$, there is a power constraint of $\mathrm{P}$ joules/symbol, and $\mathrm{E}\left[\left.\mathrm{h}[\mathrm{m}]\right|^{2}\right]=1$ is assumed for normalization. Hence $\mathrm{SNR}=\mathrm{P} / \mathrm{N}_{0}$ is the average received SNR [1].

\section{B. Uplink fading channel}

Let us now include fading. Consider the complex baseband representation of the uplink flat fading channel with K users:

$$
\mathrm{y}[\mathrm{m}]=\sum \mathrm{h}_{\mathrm{k}}[\mathrm{m}] \mathrm{x}_{\mathrm{k}}[\mathrm{m}]+\mathrm{w}[\mathrm{m}],
$$

where $\left\{\mathrm{h}_{\mathrm{k}}[\mathrm{m}]\right\}_{\mathrm{m}}$ is the fading process of user $\mathrm{k}$. We assume that the fading processes of different users are independent of each other and $E\left[\left|h_{k}[\mathrm{~m}]\right|^{2}\right]=1$. Here, we focus on the symmetric case when each user is subject to the same average power constraint, $\mathrm{P}$, and the fading processes are identically distributed [1].

\section{Effect of fading channel}

Generally in telecommunication the fading channel/multipath propagation is the main reason for Intersymbol Interference (ISI). In telecommunication, intersymbol interference (ISI) is a form of distortion of a signal in which one symbol interferes with subsequent symbols. This is an unwanted phenomenon as the previous symbols have similar effect as noise, thus making the communication less reliable. ISI is usually caused by multipath propagation or the inherent non-linear frequency response of a channel causing successive symbols to "blur" together. The presence of ISI in the system introduces errors in the decision device at the receiver output. Therefore, in the design of the transmitting and receiving filters, the objective is to minimize the effects of ISI, and thereby deliver the digital data to its destination with the smallest error rate possible. Ways to fight intersymbol interference include adaptive equalization and error correcting codes [1].

\section{Remedy of the effect of fading channel}

In the fading channel generally the ISI is caused by creating the interference between the consecutive symbols. To eliminate this problem the Viterbi equalizer is used in GSM system and Rake receiver is used in CDMA system [1]. To avoid the ISI we proposed a new OFDM symbol structure that is given below. The extra parameter TTL (Time to leave) is added in the OFDM Symbol.

\section{Proposed Method, Simulations ANd Results}

Proposed OFDM packet format, Ns-2 simulation model, and algorithm have been introduced to overcome the problem. Time-to-Live (TTL) is a value in an Internet Protocol (IP) packet that tells a network router whether or not the packet has been in the network too long and should be discarded. In other words, it specifies the number of router hops the packet is yet allowed to travel before it must be discarded or returned. Time-to-Live is a field in the IP header of a packet and is defined to be a timer limiting the lifetime of a datagram. It is an 8-bit field and the units are seconds. The Time-to-Live (TTL) field of the IP header is defined to be a timer limiting the lifetime of a datagram. It is an 8-bit field and the units are seconds. Each router (or other module) that handles a packet MUST decrement the TTL by at least one, even if the elapsed time was much less than a second. Since this is very often the case, the TTL is effectively a hop count limit on how far a datagram can propagate through the Internet which is shown in fig. 1.

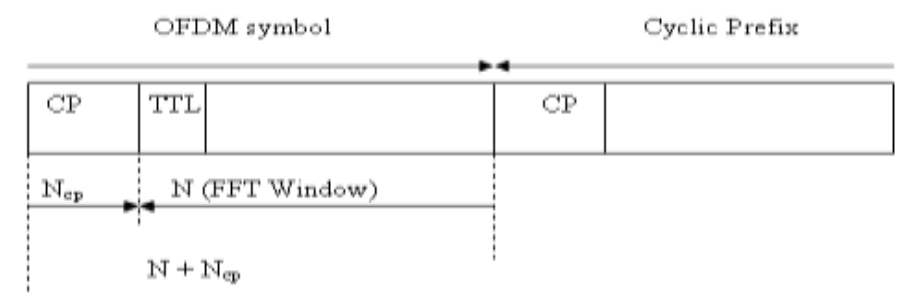

Fig.1 OFDM packet format using TTL

It is shown in Fig. 2 that from node 0, data is being sent to destination node 10. Path 0-3-6-9-10 has been confirmed for packet propagation rather than other path while they are going to be eliminated. In this experiment, every node carries the TTL value and do perform correctly. To conduct the simulation perfectly, algorithm of TTL script has also been considered in the following.

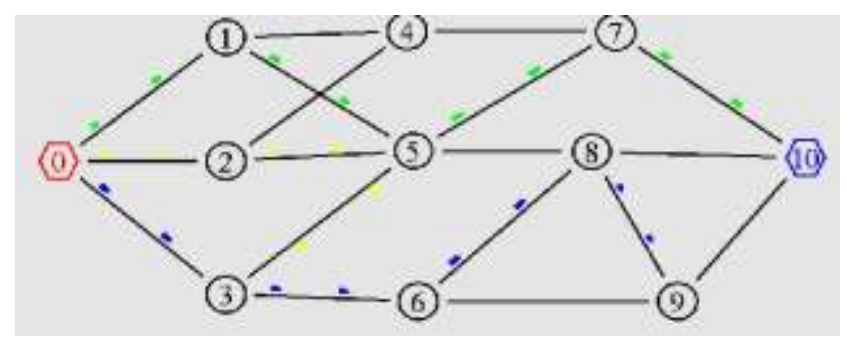

Fig.2 Simulation topology 
TCL Script Algorithm:

\section{Channel/WirelessChannel}

Propagation/TwoRayGround Phy/WirelessPhy

MAC/802_11

Queue/DropTail/PriQueue

\section{LL}

Antenna/OmniAntenna
[Numerical Value]

[Numerical Value]

\section{DSDV}

\section{[Numerical Value] \\ [Numerical Value] \\ [Numerical Value]}

System trade-offs are fundamental to all digital communication designs. It is important that the system performance may include to minimize probability of bit error and also to minimize required system bandwidth. Trade-off can be viewed as changes in the operating point on one of the curves or as changes in the operating point from one curve to another curve of the family. Movement of the operating point can be viewed as trading off between $\mathrm{P}_{\mathrm{B}}$ and $\mathrm{E}_{\mathrm{b}} / \mathrm{N}_{0}$ performances (with bandwith (W) fixed). Similarly movement can be viewed as trading off between $\mathrm{P}_{\mathrm{B}}$ and $\mathrm{W}$ performances (with $\mathrm{E}_{\mathrm{b}} / \mathrm{N}_{0}$ fixed). Also movement can be viewed as trading off between $W$ versus $E_{b} / N_{0}$ performances (with $\mathrm{P}_{\mathrm{B}}$ fixed). Simulation results based on the proposed method have been evaluated in the following figure.

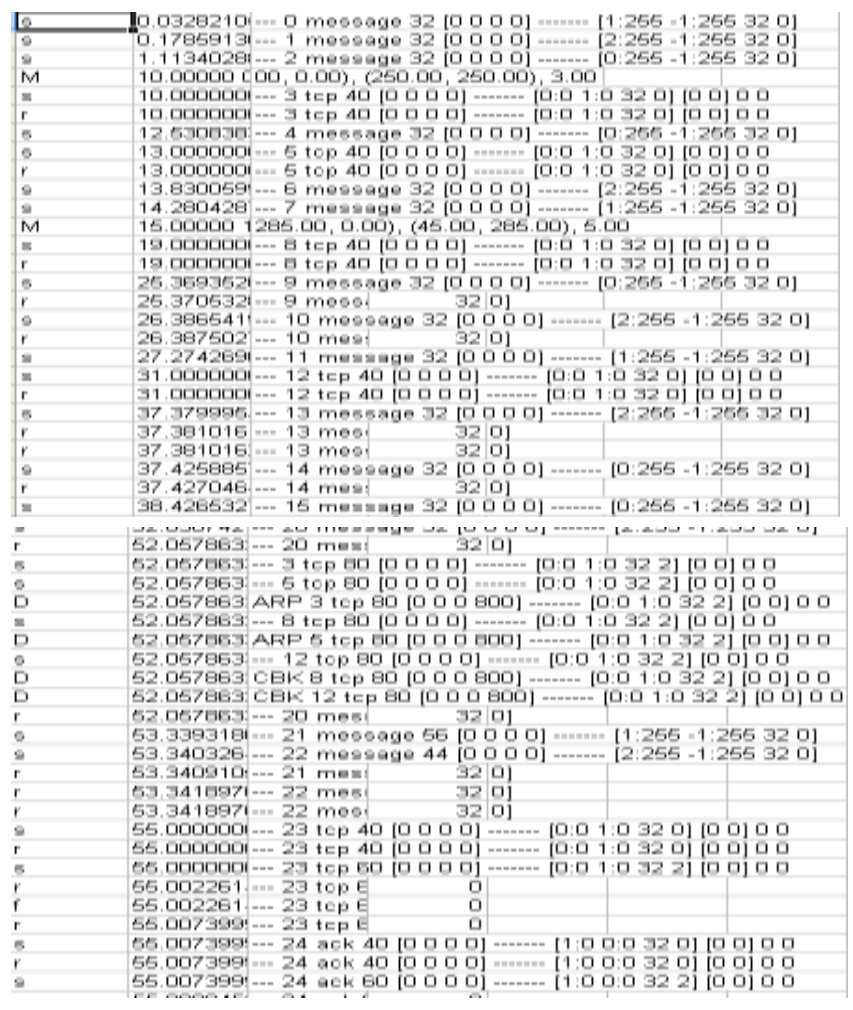

$$
\begin{array}{ll}
\text { set value } & \text { \# channel type } \\
\text { set value } & \text { \# radio-propagation model } \\
\text { set value } & \text { \# network interface type }
\end{array}
$$

set value \# MAC type set value \# interface queue type

set value \# Link Layer type

set value \# antenna model

set value \# Max Packet in interface queue

set value \# Number of mobile nodes

set value \# Routing Protocol.

set value \# X dimension of topography

set value \# Y dimension of topography

set value \# time of simulation End.

Fig. 3: Contents of the packet

During the experiment, 20898 packets have been sent on multipath fading channel and 21758 packets have been received, and 31 packets have been dropped which is mentioned in fig.3. Here the redundant bits are appeared in the receiver and movement can be viewed as trading off between $W$ versus $E_{b} / N_{0}$ performances (with $P_{B}$ fixed). From the fig. 4 , movement along points $\mathrm{B}$ to $\mathrm{D}$ can be viewed as trading off between $\mathrm{P}_{B}$ and $\mathrm{E}_{b} / \mathrm{N}_{0}$ performance (with bandwidth (W) fixed). But the proposed model, movements along points $\mathrm{B}$ to $\mathrm{C}$ can be viewed as trading off between $\mathrm{W}$ versus $\mathrm{E}_{\mathrm{b}} / \mathrm{N}_{0}$ performances (with $\mathrm{P}_{\mathrm{B}}$ fixed). Error-correction coding needs redundancy. If we assume that the system is a real-time communication system (such that the message may not be delayed), the addition of redundant bits dictates a faster rate of transmission, which of course means more bandwidth [1]. 


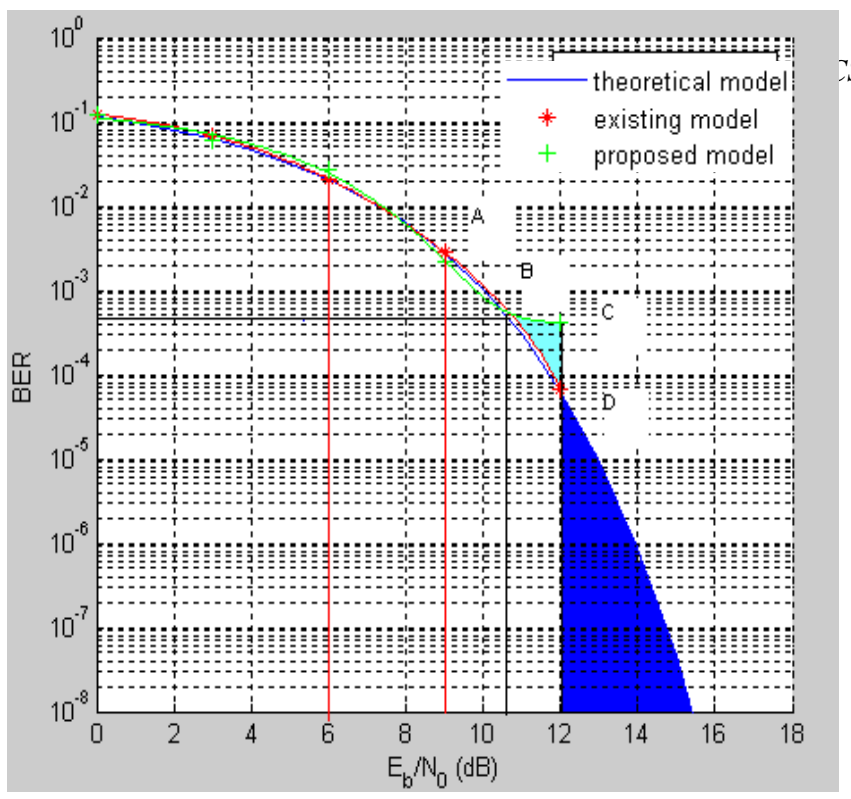

Fig.4: BER performance of the proposed model

This result ensures that due to the elimination effect of ISI and ICI, the bits of redundancy have been received and no bits are absolutely quite discarded.

\section{CONCLUSIONS}

In this paper, BER performance by considering TTL rather than effects of the ICI and ISI on the performance of OFDM systems in a time-varying multipath fading channel has been analyzed. ISI and ICI have almost totally been eliminated by considering the TTL and it is showed that the BER performance has greatly improved. To verify the result, the performance of the proposed system was examined by analysis and simulation in NS2. And it is found that the proposed improved BER performance of OFDM is better than the existing technique.

\section{REFERENCES}

[1] Bernard Sklar (2003) "Digital Communication”, ISBN 817808-373-6.

[2] Van Duc Nguyen, Kuchenbecker, H.-P. (2002) "Intercarrier and Intersymbol Interference Analysis of OFDM Systems On TimeInvariant Channels" Indoor and Mobile Radio Communications.

The 13th IEEE International Symposium on Volume 4, 15-18 Sept. 2002 Page(s):1482 - 1487 vol.4.

[3] Cheol-Jin Park; Gi-Hong Im (Sept. 2004) "Efficient DMT/OFDM Transmission with Insufficient Cyclic Prefix" Communications Letters, IEEE Volume 8, Issue 9, Page(s):576 578.

[4] Yun Hee Kim; Iickho Song; Hong Gil Kim; Taejoo Chang; Hyung Myung Kim (Sept. 1999) "Performance Analysis of a Coded OFDM System in Time-Varying Multipath Rayleigh Fading Channels", Vehicular Technology, IEEE Transactions on Volume 48, Issue 5, Page(s):1610 - 1615.

[5] Wei Zhong; Zhigang Mao (May 2006) "Efficient TimeDomain Residual ISI Cancellation for OFDM-Based WLAN Systems", Consumer Electronics, IEEE Transactions on Volume 52, Issue 2, Page(s):321 - 326.

[6] Suzuki, N.; Uehara, H. Yokoyama, M. (2002) “A NEW OFDM DEMODULATION METHOD TO REDUCE INFLUENCE OF ISI DUE TO LONGER DELAY THAN GUARD INTERVAL" Communication Systems. ICCS 2002. The 8th International Conference on Volume 1, 25-28 Nov. 2002 Page(s):239 - 244 vol.1.
SA) International Journal of Advanced Computer Science and Applications,

Vol. 1, No. 4, October 2010

[7] Minn, H.; Zeng, M.; Bhargava, V.K. (July 2000) "On Timing Offset Estimation for OFDM Systems", Communications Letters, IEEE, Volume 4, Issue 7, Page(s):242 - 244.

[8] Shaoping Chen; Cuitao Zhu (Feb. 2004) "ICI and ISI Analysis and Mitigation for OFDM Systems with Insufficient Cyclic Prefix in Time-Varying Channels" Consumer Electronics, IEEE Transactions on, Volume 50, Issue 1, Page(s):78 - 83.

[9] Klenner, P.; Kammeyer, K.-D. (22-25 April 2007) "Spatially Interpolated OFDM with Channel Estimation for Fast Fading Channels" Vehicular Technology Conference, 2007. VTC2007Spring. IEEE $65^{\text {th }}$ Page(s): $2455-2459$.

[10] Wei Zhong; Zhigang Mao (May 2006) "Efficient TimeDomain Residual ISI Cancellation for OFDM-Based WLAN Systems" Consumer Electronics, IEEE Transactions on Volume 52, Issue 2, Page(s):321 - 326.

[11] Dah-Chung Chang (Dec. 2008) "Effect and Compensation of Symbol Timing Offset in OFDM Systems With Channel Interpolation" Broadcasting, IEEE Transactions on Volume 54, Issue 4, Page(s):761 - 770 